\title{
Statistical analysis of exon lengths in various eukaryotes
}

\author{
Alexander Kaplunovsky' \\ Anatoliy Ivashchenko ${ }^{2}$ \\ Alexander Bolshoy' \\ 'Department of Evolutionary and \\ Environmental Biology, Genome \\ Diversity Center, Institute of \\ Evolution, University of Haifa, Israel; \\ ${ }^{2}$ Department of Biotechnology, \\ Biochemistry, Plant Physiology, \\ Al-Farabi Kazakh National \\ University, Kazakhstan
}

This article was published in the following Dove Press journal:

Open Access Bioinformatics

I 8 January 201 I

Number of times this article has been viewed
Purpose: The principal goals of this research were to investigate correlations between certain properties of exons in a gene (ie, between exon density and the corresponding protein length) and to compare genomic trees obtained with different approaches of clustering based on exonic parameters. The aim was a better understanding of exon-intron structures and their origin and development. The exon-intron structures of eukaryote genes are quite different from each other, and the evolution of such structures raises many problematic questions. As a preliminary attempt to address some of these questions, we performed a statistical analysis of gene exon-intron structures.

Methods: Taking whole genomes of eukaryotes, we went through all the protein-coding genes in each chromosome separately and calculated the portion of intron-containing genes and average values of the net length of all the exons in a gene, the number of the exons, and the average length of an exon. Comparing those chromosomal and genomic averages, we developed a technique of clustering based on characteristics of the exon-intron structure. This technique of clustering separates different species, grouping them according to eukaryote taxonomy.

Conclusion: Our conclusion is that the best approach is based on distances among four principal components obtained by factor analysis and followed by application of clustering algorithms, such as neighbor-joining, $k$-means, and partitioning around medoids.

Keywords: comparative genomics, exon-intron structure, eukaryotic clustering, principal component analysis

\section{Introduction}

It is no secret that people are fond of classifying things. Genomics is no exception. A lot of methods exist in comparative genomics that can be used for the purpose of genome classification. ${ }^{1}$ The objective of cluster analysis is to divide objects into clusters in a way that similarity among the items belonging to the same group is higher than similarity among items belonging to distinct groups. In this study, we intend to show that genome clustering based on exon-intron structural characteristics is essentially accurate and reliable and expands on the results of previous studies. ${ }^{2,3}$ This kind of clustering neither supports a widely accepted taxonomy nor argues against it. Uncovering and further analyzing the exon-intron structural properties that unify or distinguish genomes in the clustering procedure improve our understanding of the nature and evolutionary history of splicing.

One of the greatest enigmas of eukaryotic genome evolution is the widespread existence of introns. Introns have been detected in the genes of viruses,
Correspondence: Alexander Bolshoy Department of Evolutionary and Environmental Biology, University of Haifa, Haifa 39105, Israel

$\mathrm{Tel}+97248240382$

Fax +97248240382

Email bolshoy@research.haifa.ac.il
Open Access Bioinformatics 20I I:3 I-I5

which permits unrestricted noncommercial use, provided the original work is properly cited. 
chloroplasts, and mitochondria of both lower and higher eukaryotes. This study focuses on the most important type of introns, ie, the spliceosomal introns of nuclear-encoded protein genes. Here we survey some of the properties of the exon-intron structure of these genes in almost all completely sequenced eukaryotic genomes. Net and averaged exonic lengths are among the attributes considered in this study.

The exon and intron lengths vary across a broad range. ${ }^{4-8}$ Statistical analyses of exon and intron lengths have been performed several times on different sets of eukaryotes. ${ }^{2,3,5,8-15}$

Previously, we have shown some genome-specific features of the exon-intron organization of eukaryotic genes using a limited set of genomes from different kingdoms. ${ }^{2}$ We have shown that the most general feature found in all genomes is a positive correlation between the number of introns in a gene and the corresponding protein length (ie, the net length of all the exons of the gene). In addition, we have shown that the average exon length correlates negatively with the average number of exons. Recently, analyses of patterns of exon-intron architecture variation brought Zhu et al to the same conclusion. ${ }^{16}$ One of their main observations was a decrease in average exon length as the total exon numbers in a gene increased. Although the laws of exon-intron statistics appeared to be quite general, many of the correlation parameters were genome-specific.

Intron density, which is the average number of introns per gene, is an evolutionary riddle. At first, it was thought that one could simply predict intron density from organism complexity. Initial studies supported this hypothesis, ie, Homo sapiens has 8.1 introns per gene on average, ${ }^{17}$ Caenorhabditis elegans has $4.7,{ }^{18}$ Drosophila melanogaster has 3.4, ${ }^{19}$ and Arabidopsis thaliana has 4.4. ${ }^{20}$ In contrast, unicellular species were found to have fewer introns per gene. ${ }^{21}$ However, further studies found significantly higher intron densities in many unicellular species, ${ }^{15,22}$ and intron densities in Basidiomycetes and Zygomycete fungi appeared to be among the highest known for eukaryotes (4-6 per gene). ${ }^{23,24}$ Diversity in intron densities among fungal genomes makes them extremely attractive for exploring possible answers to questions concerning exon-intron structure evolution. Indeed, fungi display a wide diversity of gene structures, ranging from less than one intron per gene for yeasts to approximately 1-2 introns per gene, on average, for many recently sequenced lower fungi (including the organisms in this study) and to roughly 5.5 introns per gene on average for some Basidiomycetes (eg, Cryptococcus).
Following the genome sequencing of several lower eukaryotes, it has become possible to examine exon-intron statistics with sufficiently large samples of genes. The purpose of our recent publication ${ }^{3}$ was to determine the most appropriate approach to classify fungal chromosomes according to simple exon-intron statistics. We tested a few clustering techniques measuring distances among the chromosomes in different ways. As a result of our analysis, we commented on the consistent similarity of the partitions, resulting from different clustering methods. Clustering results ${ }^{3}$ obtained with scaled and normalized Euclidean distances appeared to be sufficiently similar. The principal components-based clustering method, the principal directions divisive partitioning method, and the neighbor-joining algorithm produced very similar clustering results. Therefore, we propose techniques of clustering that are able to distinguish between chromosomes of different species with satisfactory results. The addition of regression parameters to averaged chromosomal parameters improves the resolution of clustering.

There is a mixture of different chromosomal characteristics in exon-intron organization. In this study, similar to our previous publications, we considered only pure exonic properties and, additionally, proportions of intron-containing genes among all protein-coding genes. We calculated and compared exonic properties, including exon densities, average exon lengths, and average net exon lengths. In this study we investigated the correlation between the number of exons in a gene (exon density) and the corresponding protein length; compared intragenomic variation with intergenomic variance of exon densities, average exon lengths, and average net exon length; compared genomic trees obtained using different approaches of clustering based on exonic parameters; and paved a road for further evolutionary in silico research of exon-intron structure and its origins and development.

\section{Methods}

\section{Data set}

The nucleotide sequences of 322 chromosomes of 32 species presented in Table 1 were obtained from the database of the Eukaryotic Genome Sequencing Projects (http://www.ncbi. nlm.nih.gov/genomes/leuks.cgi). Gene annotations were used to calculate genic statistical properties. A standard gene annotation looks like the following annotation of a randomly chosen gene, NCU08052.1 of Neurospora crassa:

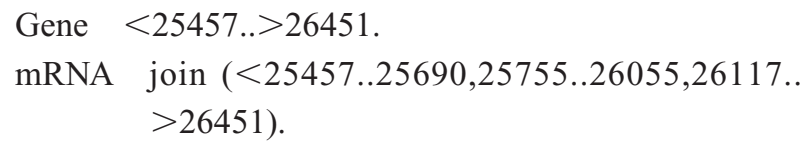


Coding sequence join (25457..25690,25755..26055, 26117..26451).

The annotation means the first exon of this gene starts somewhere upstream of position 25457 , and the last exon of the gene ends somewhere downstream of position 26451. For the purposes of this study, the term "exons" refers to "coding parts of exons". In other words, only those introns within coding sequences and exons without untranslated regions were used for analysis. The data related to coding parts of exons are taken from coding sequence lines. For example, the coding sequence of NCU08052.1 consists of three "exons" (25457:25690, 25755:26055, and 26117:26451) with lengths of $234 \mathrm{bp}, 301 \mathrm{bp}$, and $335 \mathrm{bp}$, respectively. The length of the gene is greater than $995 \mathrm{bp}$, the number of exons is equal to 3, the net length of the exons (the protein size in bp) is equal to 870 , and the average exon length is equal to 290 .

\section{Exon-intron structure and statistical parameters}

Each gene was assigned three gene-related exonic values, ie, the net length, $L_{e x}$, of all its exons, the number, $N_{e x}$, of those exons, and an average exon length, $A_{e x}$ :

$$
A_{e x}=\frac{L_{e x}}{N_{e x}}
$$

For each chromosome of each genome, several absolute and averaged chromosomal characters were calculated. In addition to the three averaged characteristics of exons, the average net length, $l_{e x}$, of all the exons in a gene per chromosome, the average number, $n_{e x}$, of the exons in a gene per chromosome, the average exon length, $a_{e x}$, per chromosome, and the proportion of intron-containing genes, $p_{c}$, as a relevant attribute were calculated. It should be

Table I List of processed species and their chromosomes

\begin{tabular}{|c|c|c|c|c|c|}
\hline $\begin{array}{l}\text { Kingdom/ } \\
\text { supergroup }\end{array}$ & Phylum & Class & Organism & Abbreviation & Chromosomes (n) \\
\hline \multirow[t]{5}{*}{ Animalia } & Arthropoda & Insecta & Drosophila melanogaster & DM & 6 \\
\hline & Chordata & Mammalia & Canis familiaris & $\mathrm{CF}$ & 19 \\
\hline & & & Homo sapiens & HS & 10 \\
\hline & & & Mus musculus & $M M$ & 10 \\
\hline & Nemata & Caenorhabditis & Caenorhabditis elegans & $\mathrm{CE}$ & 6 \\
\hline \multirow[t]{15}{*}{ Fungi } & Ascomycota & Ascomycetes & Neurospora crassa & NC & 7 \\
\hline & & Eurotiomycetes & Aspergillus fumigatus & $\mathrm{AF}$ & 8 \\
\hline & & Saccharomycotina & Candida glabrata & CG & 13 \\
\hline & & & Debaryomyces hansenii & $\mathrm{DH}$ & 7 \\
\hline & & & Eremothecium gossypii & EG & 7 \\
\hline & & & Kluyveromyces lactis & $\mathrm{KL}$ & 6 \\
\hline & & & Pichia stipitis & PS & 8 \\
\hline & & & Saccharomyces cerevisiaei & SC & 16 \\
\hline & & & Yarrowia lipolytica & YL & 6 \\
\hline & & Sordariomycetes & Gibberella zeae & $\mathrm{GZ}$ & 4 \\
\hline & & & Magnaporthe grisea & MG & 7 \\
\hline & & Taphrinomycotina & Schizosaccharomyces pombe & SP & 3 \\
\hline & Basidiomycota & Agaricomycotina & Cryptococcus neoformans & $\mathrm{CN}$ & 14 \\
\hline & & Ustilaginomycotina & Ustilago maydis & UM & 23 \\
\hline & Microsporidia & Apansporoblastina & Encephalitozoon cuniculi & $\mathrm{EC}$ & 11 \\
\hline \multirow[t]{2}{*}{ Plantae } & Magnoliophyta & Liliopsida & Oryza sativa & OS & 12 \\
\hline & & Magnoliopsida & Arabidopsis thaliana & $A D$ & 5 \\
\hline \multirow[t]{2}{*}{ Plantae/Viridiplantae } & Chlorophyta & Prasinophyceae & Micromonas sp. RCC299 & MS & 17 \\
\hline & & & Ostreococcus_lucimarinus & OL & 21 \\
\hline Protista/ & Ciliophora & Ciliatea & Paramecium tetraurelia & PT & 1 \\
\hline \multirow[t]{3}{*}{ Chromalveolata } & Apicomplexa & Aconoidasida & Plasmodium falciparum & PF & 14 \\
\hline & & & Plasmodium knowlesi & PK & 14 \\
\hline & & & Theileria annulata & TA & 3 \\
\hline \multirow[t]{2}{*}{ Protista/Chromista } & Cryptophyta & Cryptophyceae & Guillardia theta & GT & 3 \\
\hline & & & Hemiselmis anderenii & $\mathrm{HA}$ & 3 \\
\hline Protista/Protozoa & Euglenozoa & Kinetoplastea & Leishmania braziliensis & LB & 35 \\
\hline Protista/Rhizaria & Cercozoa & Chlorarachniophycea & Bigelowiella natans & $\mathrm{BN}$ & 3 \\
\hline Total & & & & & 322 \\
\hline
\end{tabular}


mentioned that $a_{e x}$ is the mean of the $A_{e x}$ values of individual genes per chromosome:

$$
a_{e x}=\frac{1}{n} \sum_{1}^{n} A_{e x}
$$

where $n$ denotes a number of genes in the chromosome here. The measure $a_{e x}$ defined in this is different from the average length, $\bar{a}_{e x}$, of all the exons in the chromosome, regardless of which gene(s) they belong to. The $\bar{a}_{e x}$ is calculated as the total length of all exons in a chromosome divided by the total number of all exons in a chromosome. ${ }^{7}$ The $a_{e x}$ usually have significantly larger values than the $\bar{a}_{e x}$ because an average length of $i$-th exon exponentially decreases with an index, $i .^{25}$

We also calculated species-averaged exon parameters, ie, $N_{g}$ (total number of genes per genome), $A N_{e x}$ (average number of exons in a gene per genome), $A L_{e x}$ (average net length of all exons in a gene per genome), $A A_{e x}$ (average exon length in a gene per genome), $A N 1_{e x}$ (average number of exons in an intron-containing gene per genome), $A L O_{e x}$ (average length of an intronless gene per genome), $A L 1_{e x}$ (average net length of all exons in an intron-containing gene per genome), and $P_{g}$ (proportion of intron-containing genes in a genome in percent).

\section{Distances between pairs of genomes}

One of our goals was to cluster genomes using exon-intron structure parameters. We used distance-based methods of clustering, so had to define a method for distance measurement. The distance between a pair of genomes was calculated as the distance between vectors constructed from several standardized parameters defined above. The vector $\bar{x}_{r}$ of genomic parameters related to genome $r$ consists of $\left(A N_{e x}, A L_{e x}, A A_{e x}\right.$, $\left.A N 1_{e x}, A L 1_{e x}, A L 0_{e x}\right)$, and is equal to

$\bar{x}_{r}=\left\{\frac{j_{e x, r}-\mu_{j}}{\sigma_{j}}\right\}, j \in\left\{A N_{e x}, A L_{e x}, A A_{e x}, A N 1_{e x}, A L 1_{e x}, A L 0_{e x}\right\}$,

where $\mu_{j}$ is the mean value of a genomic parameter $j$ and $\sigma_{j}$ is its standard deviation.

Having extracted these parameters, our next task was to find an appropriate dissimilarity measure, $d$, such that $d\left(x_{r}, x_{s}\right)$ is small if $x_{r}$ and $x_{s}$ are close. The simplest dissimilarity measure is a normalized (standardized) Euclidean distance:

$$
d\left(x_{r}, x_{s}\right)=\sqrt{\sum_{k=1}^{K}\left(\bar{x}_{r, k}-\bar{x}_{s, k}\right)^{2}}
$$

\section{Clustering of genomes}

A few popular algorithms were used to cluster all 32 genomes. First of all, the well known neighbor-joining algorithm $^{26}$ was used. Using neighbor-joining, a tree that does not assume an evolutionary clock was constructed, and therefore, in effect, an unrooted tree results. We used the Neighbor of Phylip program package from the University of Washington (http://evolution.genetics.washington.edu/ phylip/doc/neighbor.html), which is an implementation of neighbor-joining. Matrices of standardized distances between all pairs of chromosomes were exported to the Neighbor program. The output file was drawn by the TreeView program of Professor Rod Page (http://taxonomy.zoology.gla.ac.uk/ $\mathrm{rod} /$ treeview.html). We also used well known $k$-medoid clustering by partitioning around medoids and $k$-means algorithms. The $k$-medoids and $k$-means algorithms are described elsewhere. ${ }^{1}$

\section{Analyses of structural-functional organization of the system}

One-way analysis of variance (ANOVA) was used to test for differences in exon-intron structure between several groups of species. We also used factor analysis as an integral statistical method, affording an opportunity to define and evaluate the structural-functional organization of the system. We chose principal components analysis as one of the techniques for factor analysis. This method produces a set of eigenvectors calculated from the matrix of correlations between parameters where each set represents a causal connection of elements. It is important to note that by using the technique of principal components analysis, all factors become orthogonal and are caused by different properties of the system.

\section{Results}

The aforementioned chromosomal characteristics $\left(n_{e x}, l_{e x}, a_{e x}\right.$, $\left.p_{c}, l 0_{e x}, n 1_{e x}, l 1_{e x}\right)$ were calculated for all 322 chromosomes. As an illustration, the values of these characteristics for a randomly selected unicellular organism, Plasmodium knowlesi, are given in supplementary Table S1. Every column in Table S1 contains indistinguishable parameters. The intragenomic variation was found to be rather small for other unicellular organisms as well, as shown with fungi. ${ }^{3}$

The results of the one-way ANOVA test for differences in the first three parameters, $n_{e x}, l_{e x}$, and $a_{e x}$, of chromosomes of all genomes are presented in Table 2. In general, we found intragenomic variation in $l_{e x}$ and $a_{e x}$ to be quite small for almost all unicellular organisms, and this was significant in $n_{e x}, l_{e x}$, and $a_{e x}$ for Plantae and Animalia (especially for 
Table 2 Results of one-way ANOVA test for differences in parameters between chromosomes for several species

\begin{tabular}{|c|c|c|c|c|}
\hline Organism & Kingdom/supergroup & $n_{e x}$ & $I_{\mathrm{ex}}$ & $a_{e x}$ \\
\hline$A D$ & Plantae & $0.006 * *$ & $0.000 * * *$ & $0.007 * *$ \\
\hline $\mathrm{AF}$ & Fungi & 0.211 & 0.097 & 0.431 \\
\hline $\mathrm{BN}$ & Protista/Rhizaria & 0.591 & 0.790 & 0.193 \\
\hline $\mathrm{CE}$ & Animalia & $0.000 * * *$ & $0.000 * * *$ & $0.000 * * *$ \\
\hline $\mathrm{CF}$ & Animalia & $0.000^{* * * *}$ & $0.000 * * *$ & $0.000 * * *$ \\
\hline CG & Fungi & - & 0.979 & 0.976 \\
\hline $\mathrm{CN}$ & Fungi & 0.591 & 0.764 & 0.077 \\
\hline $\mathrm{DH}$ & Fungi & - & 0.190 & 0.058 \\
\hline DM & Animalia & $0.000 * * *$ & $0.000 * * *$ & $0.000 * * *$ \\
\hline $\mathrm{EC}$ & Fungi & - & 0.203 & 0.226 \\
\hline EG & Fungi & - & 0.377 & 0.423 \\
\hline GT & Protista/Chromista & - & 0.128 & 0.112 \\
\hline GZ & Fungi & $0.000 * * *$ & $0.040 * *$ & $0.000 * * *$ \\
\hline $\mathrm{HA}$ & Protista/Chromista & - & 0.599 & 0.599 \\
\hline $\mathrm{HS}$ & Animalia & $0.002^{* *}$ & 0.123 & $0.000 * * *$ \\
\hline $\mathrm{KL}$ & Fungi & - & 0.427 & 0.389 \\
\hline LB & Protista/Protozoa & - & $0.003 * *$ & $0.002^{* *}$ \\
\hline MG & Fungi & $0.045^{* *}$ & $0.014^{*}$ & 0.565 \\
\hline MM & Animalia & $0.000^{* * * *}$ & $0.000 * * *$ & $0.000 * * *$ \\
\hline MS & Plantae/Viridiplantae & $0.000 * * *$ & 0.342 & $0.002 * *$ \\
\hline NC & Fungi & $0.037^{*}$ & $0.009 * *$ & 0.947 \\
\hline OL & Plantae/Viridiplantae & $0.000 * * *$ & 0.305 & 0.863 \\
\hline OS & Plantae & $0.000 * * *$ & 0.075 & $0.000 * * *$ \\
\hline PF & Protista/Chromalveolata & 0.083 & 0.168 & 0.053 \\
\hline PK & Protista/Chromalveolata & $0.47 I$ & 0.770 & 0.548 \\
\hline PS & Fungi & 0.253 & 0.392 & 0.203 \\
\hline PT & Protista/Chromalveolata & - & - & - \\
\hline SC & Fungi & 0.692 & 0.993 & 0.985 \\
\hline $\mathrm{SP}$ & Fungi & 0.651 & 0.570 & 0.321 \\
\hline TA & Protista/Chromalveolata & $0.004^{* *}$ & 0.771 & 0.680 \\
\hline UM & Fungi & 0.309 & 0.539 & 0.366 \\
\hline YL & Fungi & - & 0.319 & 0.523 \\
\hline
\end{tabular}

Notes: *significance $0.01<P<0.05$; $*$ significance $0.001<P<0.01$; $* * *$ significance $P<0.001$; - parameters that did not pass the Levene test of homogeneity.

D. melanogaster chromosomes, with an outstanding and short chromosome 4). Table 2 shows that the sets $a_{e x}, l_{e x}$, and $n_{e x}$ in various chromosomes demonstrate significant differences. We can see that F-statistics comparing variances between and within groups of chromosomes are significant. The ANOVA method was used only for parameters that passed the Levene test of homogeneity. As can be seen, most species with a low percentage of intron-containing genes in chromosome $p_{c}$ did not pass this test for $n_{e x}$.

Problems investigated in this study included correlations between different species-averaged parameters of exonintron structure, clustering chromosomes of a few organisms belonging to the same kingdom (Protista, Plantae, and Animalia) by combinations of chromosome-averaged exonic characteristics, and clustering of all 32 organisms by combinations of species-averaged characteristics of exons.

\section{Correlations among species-averaged statistical parameters}

In Table 3, in addition to parameters averaged over all genes, there are data related to a set of "intron-containing" genes $\left(A L 1_{e x}\right)$ and to a set of "intronless" genes $\left(A L O_{e x}\right)$. In the Methods section, there are descriptions and formulae for calculations of these parameters. Some putative empiric rules may be deduced from Table 3. For example, regarding average protein lengths of intron-containing and intronless genes (net length of all exons), it seems that if there is only a small amount of intron-containing genes in a genome, such proteins are shorter on average than other proteins coded by intronless genes of the same genome. This property is especially strongly expressed for some species of fungi (Encephalitozoon cuniculi, Candida glabrata, and Kluyveromyces lactis and also exists for Eremothecium gossypii, Debaryomyces hansenii, Schizosaccharomyces pombe, and Ustilago maydis), and for three Protista species (Leishmania braziliensis, Hemiselmis anderenii, and Guillardia theta). Figure 1 shows a scatter-plot of $P_{g}$ versus a fraction of $A L O_{e x} / A L 1_{e x}$ and is obtained from Table 3. H. anderenii does not appear in Figure 1 because it has no intron-containing genes. There are three main groups of points in the plot, ie, a group of genomes with a low concentration of intron-containing genes $\left(P_{g}<10 \%\right)$, a group of genomes with a high concentration of intron-containing genes $\left(P_{g}>70 \%\right)$, and an intermediate group. The first group is mainly characterized by a striking prevalence of longer genes among intronless genes compared with intron-containing ones. We could deduce a rule that, in genomes with a low presence of intron-containing genes, such genes are coding shorter proteins. However, there is an exception to this empiric rule, ie, L. braziliensis, which has a fraction $A L 0_{e x} / A L 1_{e x}$ similar to genomes with high $P_{g}$. An empiric rule for the second group may be formulated that there is a (linear) positive correlation between a proportion of intron-containing genes in a genome and a fraction $A L 0_{e x} / A L 1_{e x}$ while values of a fraction are lower than 1. Unfortunately, we have an exception to this rule as well, ie, Bigelowiella natans, which has a surprisingly high value of the ratio $A L 0_{e x} / A L 1_{e x}$. Regarding the central group, we may say only that it has an intriguing configuration that requires further investigation.

\section{Chromosome-averaged statistical parameters}

Let us consider the average parameters $l_{e x}, n_{e x}$, and $a_{e x}$. A scatter-plot of $a_{e x}$ versus $l_{e x}$ is shown in Figure 2B for Protista and illustrates the statement made previously that 
Table 3 Species dependent exonic parameters

\begin{tabular}{|c|c|c|c|c|c|c|c|c|}
\hline Organism & Kingdom/supergroup & $A N_{\text {ex }}$ & $A L_{\text {ex }}$ & $A A_{\text {ex }}$ & $A N I_{e x}$ & $A L I_{e x}$ & $P_{g}$ & $A L 0_{\text {ex }}$ \\
\hline AD & Plantae & 5.255 & 1243 & 412 & 6.404 & 1322 & 78.65 & 951 \\
\hline $\mathrm{AF}$ & Fungi & 2.918 & 1465 & 668 & 3.453 & 1513 & 78.14 & 1289 \\
\hline $\mathrm{BN}$ & Protista/Rhizaria & 4.054 & 960 & 359 & 4.580 & 907 & 85.37 & 1142 \\
\hline CE & Animalia & 6.283 & 1284 & 213 & 6.428 & 1306 & 97.34 & 457 \\
\hline CF & Animalia & 10.697 & 1696 & 232 & I I.450 & 1762 & 92.74 & 843 \\
\hline CG & Fungi & 1.016 & 1509 & 1504 & 2.025 & 662 & 1.56 & 1522 \\
\hline $\mathrm{CN}$ & Fungi & 6.271 & 1611 & 319 & 6.445 & 1627 & 96.87 & 1123 \\
\hline $\mathrm{DH}$ & Fungi & 1.057 & 1387 & 1357 & 2.075 & 1070 & 5.41 & 1402 \\
\hline DM & Animalia & 3.914 & 1824 & 503 & 4.686 & 2007 & 81.12 & 906 \\
\hline EC & Fungi & 1.008 & |07| & 1069 & 2.143 & 438 & 0.71 & 1075 \\
\hline EG & Fungi & 1.048 & 1472 & 1452 & 2.035 & 874 & 4.58 & 1485 \\
\hline GT & Protista/Chromista & 1.033 & 939 & 930 & 2.000 & 583 & 3.29 & 952 \\
\hline GZ & Fungi & 3.261 & $|53|$ & 623 & 3.590 & 1553 & 82.04 & 1359 \\
\hline $\mathrm{HA}$ & Protista/Chromista & 1.000 & 1019 & 1019 & - & - & 0.00 & 1019 \\
\hline $\mathrm{HS}$ & Animalia & 8.868 & 1533 & 280 & 10.167 & 1656 & 85.76 & 790 \\
\hline $\mathrm{KL}$ & Fungi & 1.025 & 1418 & 1409 & 2.017 & 760 & 2.47 & 1435 \\
\hline LB & Protista/Protozoa & 1.012 & 1905 & 1882 & 2.040 & 3854 & 1.16 & 1882 \\
\hline MG & Fungi & 2.844 & 1394 & 654 & 3.480 & 1468 & 74.34 & 1179 \\
\hline$M M$ & Animalia & 8.248 & 1457 & 302 & 9.658 & 1575 & 83.53 & 848 \\
\hline MS & Plantae/Viridiplantae & 1.516 & 1488 & 1166 & 2.447 & 1636 & 34.58 & 1407 \\
\hline NC & Fungi & 2.703 & 1476 & 694 & 3.136 & 1505 & 79.73 & 1366 \\
\hline OL & Plantae/Viridiplantae & 1.279 & 1253 & 1100 & 2.344 & 1388 & 20.06 & 1222 \\
\hline OS & Plantae & 4.846 & 1237 & 440 & 6.054 & 1348 & 75.96 & 890 \\
\hline PF & Protista/Chromalveolata & 2.440 & 2238 & 1490 & 3.603 & 2131 & 55.30 & 2377 \\
\hline PK & Protista /Chromalveolata & 2.591 & 2189 & 1486 & 4.094 & 2021 & 51.43 & 2373 \\
\hline PS & Fungi & 1.408 & 1495 & 1227 & 2.551 & 1732 & 26.28 & 1409 \\
\hline PT & Protista/Chromalveolata & 3.337 & 1583 & 583 & 3.803 & 1674 & 83.37 & 1128 \\
\hline SC & Fungi & 1.055 & 1482 & 1444 & 2.035 & 1434 & 5.31 & 1485 \\
\hline SP & Fungi & 1.951 & 1413 & 1040 & 3.098 & 1305 & 45.36 & $|50|$ \\
\hline TA & Protista/Chromalveolata & 3.775 & $|58|$ & 785 & 4.964 & 1525 & 69.96 & 1716 \\
\hline UM & Fungi & I.782 & 1839 & 1439 & 3.025 & 1649 & 38.60 & 1961 \\
\hline YL & Fungi & I.I58 & 1458 & 1339 & 2.131 & 1637 & 13.92 & 1428 \\
\hline Total & & 3.121 & 1579 & 1057 & 4.204 & 1606 & 42.97 & 1417 \\
\hline
\end{tabular}

the averages of $l_{e x}$ and $a_{e x}$ were fairly similar for different chromosomes of the same species but, as a rule, rather distant for different species. Moreover, six separate groups of points may be observed in Figure 2B.

We colored all points using four colors relating to four Protista supergroups, ie, Chromalveolata (Plasmodium falciparum, P. knowlesi, Paramecium tetraurelia, and Theileria annulata), Chromista (Guillardia theta, H. anderenii), Protozoa (L. braziliensis), and Rhizaria (B. natans, see Table 1). Analyzing the contents of the groups presented in Figure 2, one can suppose that the divisions follow their taxonomy. Indeed, scatter-plots of $a_{e x}$ vs $n_{e x}$ (Figure 2A) and $a_{e x}$ vs $l_{e x}$ (Figure 2b) clearly show six separate groups of chromosomes; $B$. natans chromosomes belonging to Rhizaria form the left-most group, $G$. theta and $H$. anderenii chromosomes belonging to Chromista are located together, and Protozoa (L. braziliensis) form the third cluster. Chromosomes belonging to Chromalveolata form three clusters, according to their phylum and class, ie, Apicomplexa Plasmodium (P. falciparum and P. knowlesi), Apicomplexa Theileria (T. annulata), and a single chromosome of Paramecium (P. tetraurelia). These scatter-plots show that the three parameters $a_{e x}, n_{e x}$, and $l_{e x}$ are sufficient for successful classification of 76 chromosomes to eight unicellular organisms.

The same conclusion regarding classification mirroring the phyla taxonomy can be made following an analysis of the matching chromosomal parameters for Animalia. Scatter-plots of $a_{e x}$ versus $l_{e x}$ and $a_{e x}$ versus $n_{e x}$ for Animalia are shown in Figure 3. Points related to averages $l_{e x}$ and $a_{e x}$ were related to different chromosomes of the same species and were located quite close to one another, whereas points related to chromosomes of different species are placed distant from one another. Striking exceptions are the points associated with chromosome 4 of $D$. melanogaster and chromosome 7 of Mus musculus. These points form clusters of a single member clearly disjointed from other 


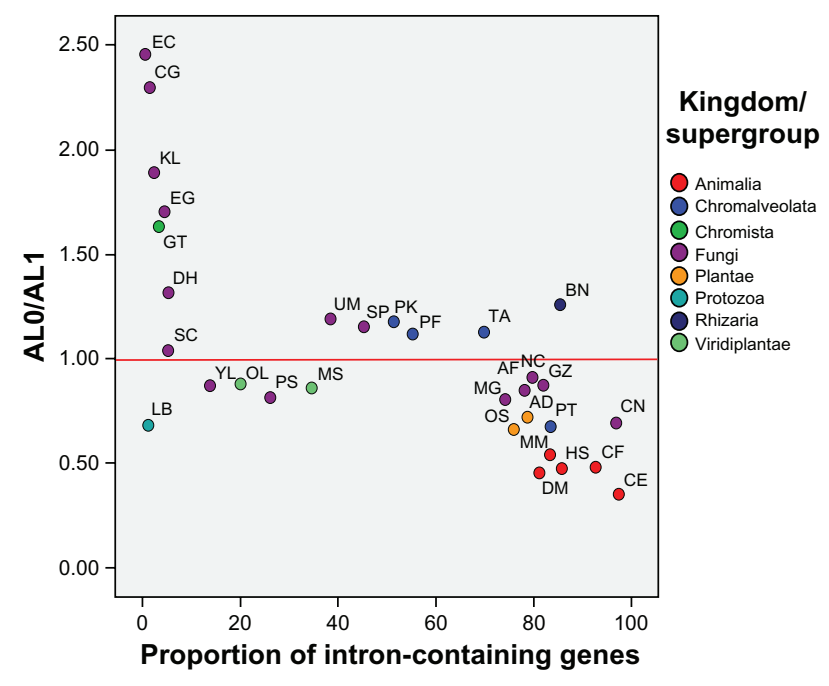

Figure I Scatter-plot of $P_{g}$ showing the proportion of intron-containing genes in a genome on the $\mathrm{x}$-axis versus the ratio between $A L O_{\text {ex }}$ (an average length of an intronless gene) and $A L I_{\text {ex }}$ (an average net length of all exons in an intron-containing gene) on the $y$-axis for all 32 genomes. The red line marks the level of equality of $A L O_{\text {ex }}$ and $A L I_{\text {ex }}$

groups. All other points form three separate groups, which may be observed in Figure 3. The two parameters $l_{e x}$ and $a_{e x}$ separately cluster five chromosomes of $D$. melanogaster in one group, six chromosomes of C. elegans in another group, and all 39 chromosomes of Canis familiaris, H. sapiens, and M. musculus in the third group.

Let us repeat our observations deduced from Figure 3 relating to the phyla. We colored all points in three colors related to three animal phyla (see Table 1), ie, Arthropoda, Chordata, and Nemata. Figure 3a presents a scatter-plot of $a_{e x}$ versus $n_{e x}$ and clearly shows three separate groups of chromosomes and two outliers. C. familiaris, $H$. sapiens, and $M$. musculus chromosomes belonging to Chordata Mammalia form the left-most group; C. elegans chromosomes belonging to Nemata Caenorhabditis appear in the second left group; and the points belonging to D. melanogaster (Arthropoda Insecta) appear in the right group. Two chromosomes, ie, DM4 (the shortest chromosome of D. melanogaster) and MM07, form two separate groups, each one with a single member. The $C$. elegans chromosomes have the greatest exon density $\left(n_{e x}\right)$ and the shortest exons $\left(l_{e x}\right)$ among all the animal chromosomes studied.

\section{Clustering of genomes by species- averaged statistical parameters}

After the relatively satisfying success of partial clustering based on only three chromosomal characteristics, our next objective was to cluster all 32 genomes. We took seven species-averaged exon parameters mentioned previously, ie, $A N_{e x}$ (average number of exons in a gene per genome), $A L_{e x}$ (average net length of all exons in a gene per genome), $A A_{e x}$ (average exon length in a gene per genome), $A N 1_{e x}$ (average number of exons in an intron-containing gene per genome), $A L O_{\text {ex }}=$ average (over a genome) length of an intronless gene, $A L 1_{e x}$ (average net length of all exons in an intron-containing gene per genome), and $P_{g}$ (proportion of intron-containing genes in a genome expressed as a percentage). The expectation was that clustering would generally follow the kingdom/supergroup/phylum classification. However, the
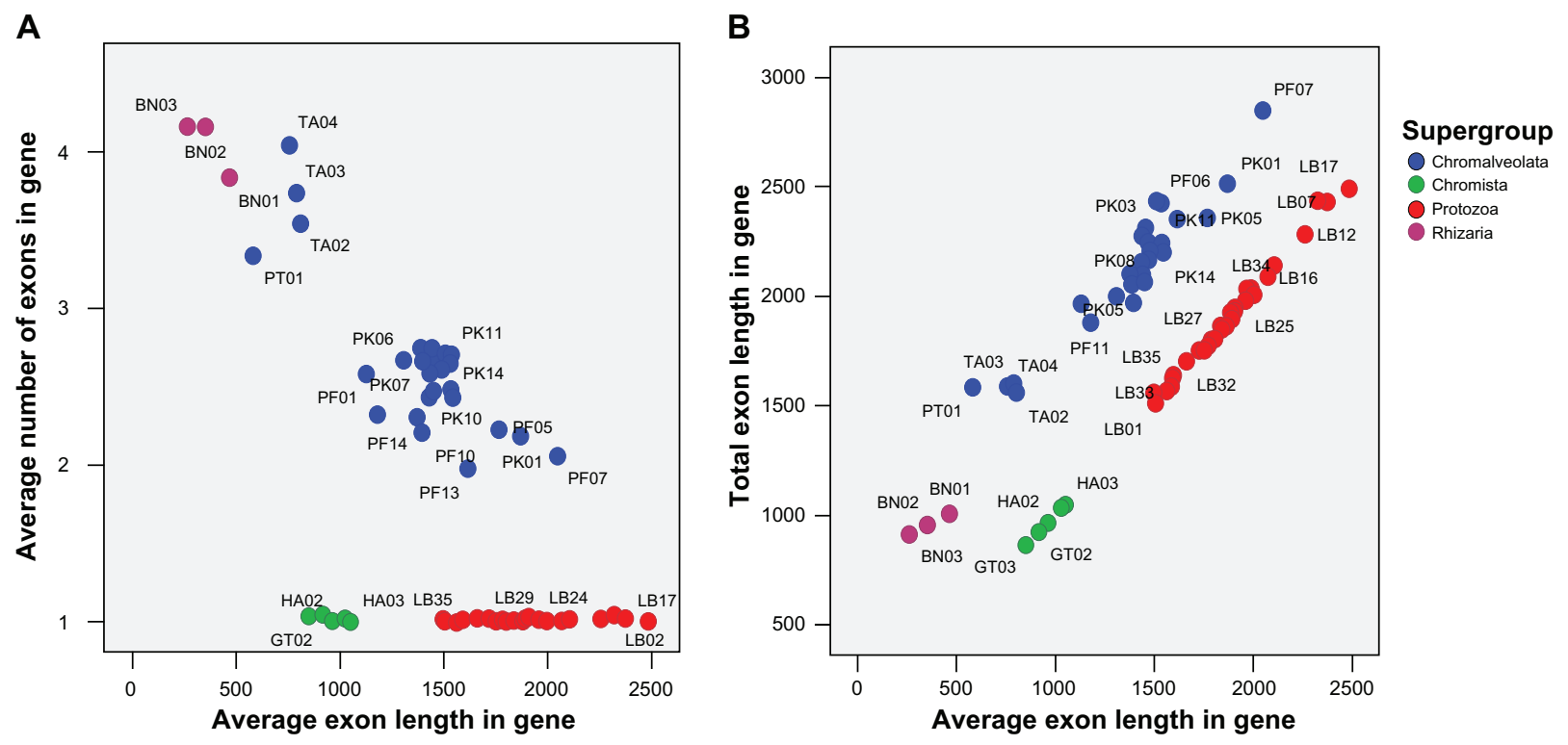

Figure 2 Scatter-plot for 76 processed chromosomes of eight Protista species, colored by four supergroups. Plot presents the average exon length per gene $a_{\text {ex }}$ ( $x$-axis) A) versus the average number of exons per gene $n_{e x}\left(y\right.$-axis) and B) versus the average net exon length per gene $I_{e x}(y$-axis). 

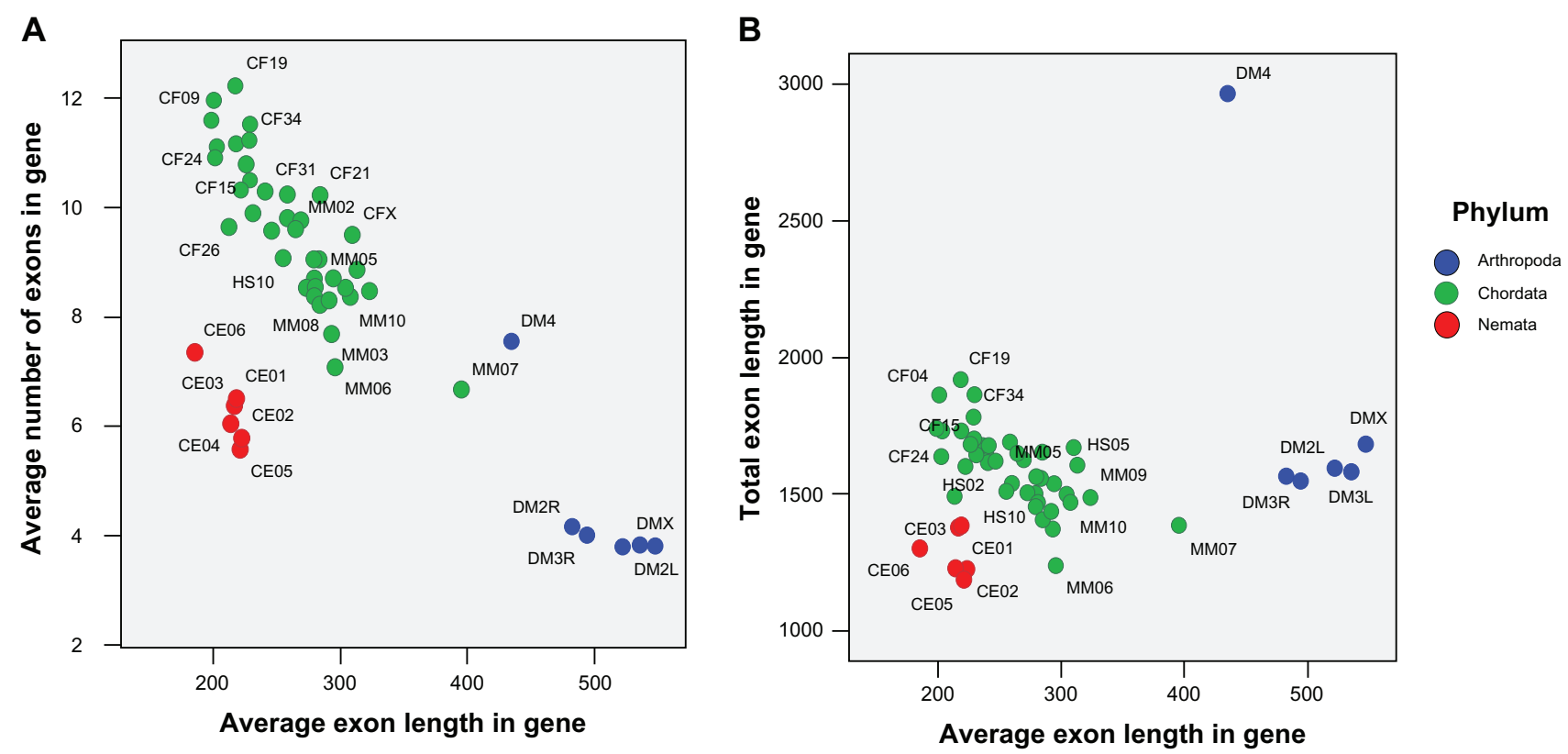

Figure 3 Scatter-plot for 59 processed chromosomes of five Animalia species, colored by three phyla. Plot presents the average exon length per gene $a_{\text {ex }}$ (x-axis) A) versus the average number of exons per gene $n_{e x}(y$-axis) and $\mathbf{B})$ versus the average net exon length per gene $l_{\text {ex }}(y$-axis).

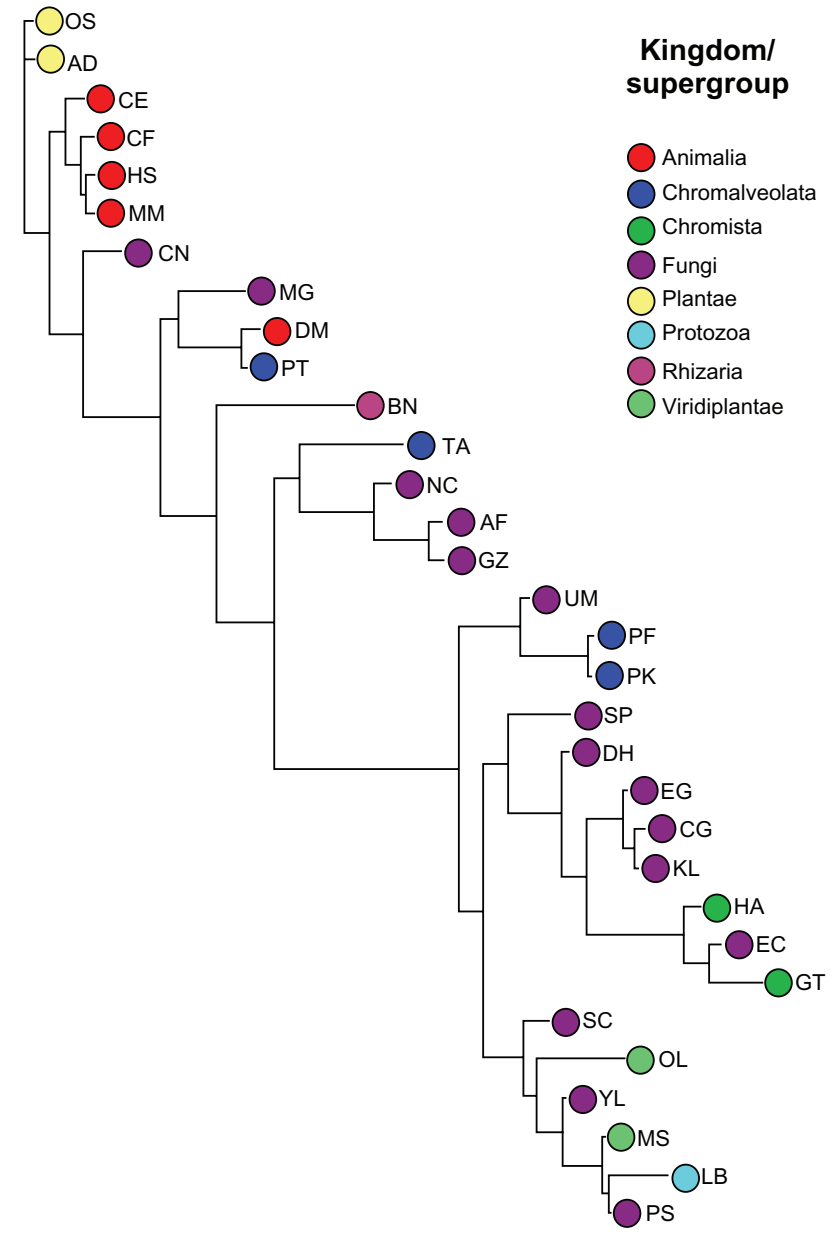

Figure 5 Dendrogram of 32 processed genomes obtained by neighbor-joining clustering technique and based on distances among four principal components obtained by factor analysis of $A N_{e x}, A L_{e x}, A A_{e x}, A N I_{e x}, A L I_{e x}$, and $A L O_{e x}$. results were poor (data not shown). Assuming that a peculiar relationship between a parameter $P_{g}$ and other parameters (see Figure 1) may negatively influence clustering, we excluded this parameter from further consideration.

At this point, we tried to cluster genomes of 32 different organisms using six parameters, namely $A N_{e x}, A L_{e x}, A A_{e x}$, $A N 1_{e x}, A L 1_{e x}$, and $A L 0_{e x}$. As a first stage, we applied neighbor-joining clustering using standardized distances among the vectors $\left(A N_{e x}, A L_{e x}, A A_{e x}, A N 1_{e x}, A L 1_{e x}, A L 0_{e x}\right)$ and applying the Neighbor program. The dendrogram presented in Figure 4 was drawn by the TreeView program. As one can see, some organisms of the same kingdom/supergroup are distributed compactly along the tree. Nevertheless, not all species belonging to the same class form a monophyletic cluster. Mice (M. musculus), dogs (C. familiaris), and humans ( $H$. sapiens) are located together, but flies (D. melanogaster), which form a cluster together with Protista/Chromalveolata, T. annulata, appear too far away from other Animalia. Viridiplantae species are placed distantly, and Protista are distributed along the tree in a strange manner. Such a classification, although better than the classification produced by seven parameters, cannot be considered adequate.

These discrepancies could be explained at least partially by the cross-dependencies of all the parameters considered. Therefore, the way to improve clustering is to replace these parameters by independent (orthogonal) parameters that could be obtained, eg, from results of a factor analysis of their correlation matrix as principal components. 


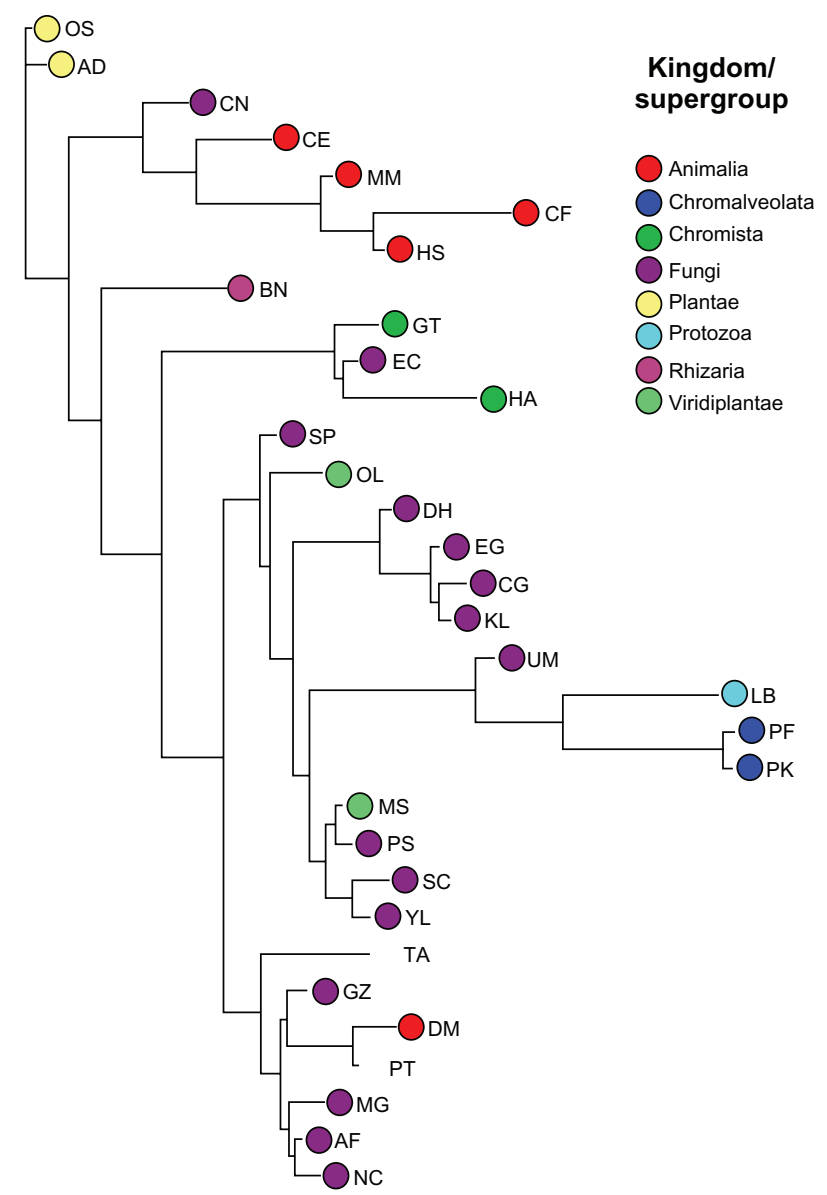

Figure 4 Dendrogram of 32 processed genomes obtained by the neighbor-joining clustering technique and based on standardized distances among parameters $A N_{e x^{\prime}}, A L_{e x^{\prime}}, A A_{e x^{\prime}}, A N I_{e x^{\prime}} A L I_{e x^{\prime}}$, and $A L O_{e x}$

The factor analysis led us to the synthesis of the following successive logical structure:

- Dividing the system into sets of "elementary" components, ie, all of the aforementioned genomic characteristics $\left(A N_{e x}, A L_{e x}, A A_{e x}, A N 1_{e x,}, A L 1_{e x}, A L 0_{e x}\right)$

- Analysis of the relationships of these components in species

- Revealing system-forming relationships

- Description of the structure of the system (model) and its properties.

As shown in Table S2, four principal components are responsible for $99.4 \%$ of the organization of the whole system, and the first two describe $86.2 \%$ of the whole variability of the system. Four principal components (Table S3) have been used in genome clustering based on neighbor-joining, $k$-means, and partitioning around medoids. Results of neighbor-joining clustering are presented in Figure 5.

There are certain improvements comparing the clustering presented in Figure 4. Viridiplantae species are placed closely, and Protista are distributed along the tree less strangely than in Figure 4. However, D. melanogaster couples with the Protista T. annulata again.

Results of $k$-means (Table S4) clustering are very similar (practically identical) to the neighbor-joining results shown earlier. These $k$-means results are shown in Table S4. Results of partitioning around medoids clustering are presented in Table S5. These results are similar to neighbor-joining results as well. However, there are some additional improvements in partitioning genomes among different clusters. In general, the results show a high consistency of partitioning, in spite of differences in clustering techniques. Careful examination of Table S5 reveals hierarchic partitioning of organisms. Interestingly, partitioning around medoids clustering is not a hierarchic algorithm and should not necessarily produce any hierarchy. In our case of application of partitioning around medoids clustering to four principal components obtained by factor analysis, a strictly hierarchic structure is produced. In fact, the $k$-medoids clustering was performed for different values of $k$ between 2 and 20, and it was observed that the clustering for a given value of $k$ is always a strict subclustering of the clustering for $k-1$. This may be interpreted as existence of an intrinsic hierarchic structure of principal components analysis data. This may, in turn, serve as additional evidence of variance in the evolutionary nature of exon-intron structure.

\section{Discussion}

The origin of introns remains a mystery, and certain questions in molecular evolution are being investigated by in silico analysis of intron-exon structures in various organisms. To facilitate such studies, while taking advantage of the burgeoning amount of sequence data now available, we undertook a statistical analysis of the exon-intron structure for nearly all completely sequenced eukaryotic genomes in order to reveal general and genome-specific features of eukaryotic genes. We went through all of the protein-coding genes in each chromosome separately and calculated the portion of intron-containing genes and average values of the net length of all the exons in a gene, the number of exons, and the average length of an exon. Furthermore, we tried to determine the most appropriate approach to classifying eukaryotic chromosomes, according to these simple exon-intron statistics.

One of the main conclusions of the studies by Kaplunovsky et $\mathrm{al}^{2}$ and Atambayeva et $\mathrm{al}^{13}$ was that a positive correlation exists between the number of introns in a gene and the length of the corresponding protein (and equivalently the net length 
of all the exons of the gene). Here, like Kaplunovsky et $\mathrm{al}^{3}{ }^{3}$ we confirmed the observation of Ivashchenko et al ${ }^{15}$ that, for all fungal genomes with a proportion of intron-containing genes higher than $30 \%$, gene size and total exon length depend on the intron number in a linear manner. The correlation problem is irrelevant for organisms with an extremely low proportion of intron-containing genes, such as yeasts, Protista/Chromista, and Protista/Protozoa.

In a previous publication, ${ }^{2}$ we reported that intragenomic variation is substantially smaller than intergenomic variance in almost all fungal genomes. In other words, we found that the laws of exon-intron statistics are specific to genomes rather than to individual chromosomes. In this respect, the similarity in exon-intron structures for dogs (C. familiaris), mice (M. musculus), and humans (H. sapiens) is so striking that intragenomic and intergenomic variances of the sets $a_{e x}, l_{e x}$, and $n_{e x}$ in various chromosomes are practically undetectable (see Table 2). A similar statement can be made regarding two plants in this study, ie, Arabidopsis and rice, and thus we confirmed the observations made by Atambayeva et al. ${ }^{13}$

Noteworthy is the similarity in the exon-intron structures of an insect, $D$. melanogaster, and a protist, T. annulata (see Table 3). Neither environmental habitat factors nor the evolutionary history of organisms provide any clue to solving the mystery of the proximity of these two genomes on the genome tree based on exon-intron characteristics. Perhaps the appearance of other eukaryotes in the data set of completely sequenced genomes will provide the answer.

The main advances of this study over previous research ${ }^{2,3,5,8-15}$ lie in the larger amount of genomes considered and the concentrated efforts made to determine the most appropriate approach for clustering based on exonic characteristics. We checked a few procedures of clustering based on exon-intron structure features averaged over intron-containing or intronless genes. As a result, we conclude that the most successful procedure should be based on distances between four principal components obtained by factor analysis and followed by application of clustering algorithms. The consistency of recovered cluster structures may be considered evidence of hidden evolutionary resemblance.

We concentrated our efforts on comparison of exonic parameters, while planning to work on intron lengths later. Clearly, the exon-intron structures of eukaryotic genes have many important parameters that we did not consider in this work, and we intend to pursue these in future research. In particular, the ratio of exon and intron lengths promises to be an important feature of a gene. In some genomes, the intron length is comparable with the exon length, ie, in unicellular eukaryotes, ${ }^{4,5}$ plants, ${ }^{5,27}$ and particular animals.$^{5-7}$ In general, introns are longer than exons in mammalian genes. ${ }^{14}$ Correlations of intronic characteristics with such genomic properties as gene density would be a goal for further research as well.

\section{Acknowledgments}

We are grateful to S Hosid and Z Volkovich for technical assistance with this research.

\section{Disclosure}

The authors report no conflicts of interest in this work.

\section{References}

1. Bolshoy A, Volkovich Z, Kirzhner V, Barzily Z. Genome clustering: From Linguistic Models to Classification of Genetic Texts. Studies in Computational Intelligence Series. Kacprzyk J, editor. Berlin, Heidelberg: Springer-Verlag; 2010.

2. Kaplunovsky A, Khailenko VA, Bolshoy A, Atambayeva SA, Ivashchenko AT. Statistics of exon lengths in animals, plants, fungi, and protists. Int J Biol Life Sci. 2009;1:139-144.

3. Kaplunovsky A, Zabrodsky D, Volkovich Z, Ivashchenko AT, Bolshoy A. Statistics of exon lengths in fungi. Open Bioinformatics $J$. 2010;4:31-40.

4. Kupfer DM, Drabenstot SD, Buchanan KL, et al. Introns and splicing elements of five diverse fungi. Eukaryot Cell. 2004;3:1088-1100.

5. Deutsch M, Long M. Intron-exon structures of eukaryotic model organisms. Nucleic Acids Res. 1999;27:3219-3228.

6. Wendel JF, Cronn RC, Alvarez I, Liu B, Small RL, Senchina DS. Intron size and genome size in plants. Mol Biol Evol. 2002;19: 2346-2352.

7. Sakharkar MK, Chow VT, Kangueane P. Distributions of exons and introns in the human genome. In Silico Biol. 2004;4:387-393.

8. Roy SW, Penny D. Intron length distributions and gene prediction. Nucleic Acids Res. 2007;35:4737-4742.

9. Naora H, Deacon NJ. Relationship between the total size of exon and introns in the protein-coding genes of higher eukaryotes. Proc Natl Acad Sci U S A. 1982;79:6196-6200.

10. Hawkins JD. A survey on intron and exon lengths. Nucleic Acids Res. 1988;16:9893-9908.

11. Kriventseva EV, Gelfand MS. Statistical analysis of the exon-intron structure of higher and lower eukaryote genes. J Biomol Struct Dyn. 1999;17:281-288

12. Ivashchenko AT, Atambayeva SA. Variation in lengths of introns and exons in genes of the Arabidopsis thaliana nuclear genome. Russ J Genet. 2004;40:1179-1181.

13. Atambayeva SA, Khailenko VA, Ivashchenko AT. Intron and exon length variation in arabidopsis, rice, nematode, and human. Mol Biol. 2008;42:312-320.

14. Ivashchenko AT, Khailenko VA, Atambayeva SA. Variation of the lengths of exons and introns in human genome genes. Russ J Genet. 2009;45:16-22.

15. Ivashchenko AT, Tauasarova MI, Atambayeva SA. Exon-intron structure of genes in complete fungal genomes. Mol Biol. 2009;43:24-31.

16. Zhu LC, Zhang Y, Zhang W, Yang SH, Chen JQ, Tian DC. Patterns of exon-intron architecture variation of genes in eukaryotic genomes. BMC Genomics. 2009;10:12.

17. Collins FS, Lander ES, Rogers J, Waterston RH. International Human Genome Sequencing Consortium: Finishing the euchromatic sequence of the human genome. Nature. 2004;431:931-945.

18. Schwarz EM, Antoshechkin I, Bastiani C, et al. WormBase: Better software, richer content. Nucleic Acids Res. 2006;34 Database Issue: D475-D478. 
19. Drysdale RA, Crosby MA, FlyBase C. FlyBase: Genes and gene models. Nucleic Acids Res. 2005;33:D390-D395.

20. Haas BJ, Wortman JR, Ronning CM, et al. Complete reannotation of the Arabidopsis genome: Methods, tools, protocols and the final release. BMC Biol. 2005;3:7.

21. Logsdon JMJ, Stoltzfus A, Doolittle WF. Molecular evolution: Recent cases of spliceosomal intron gain? Curr Biol. 1998;8:R560-R563.

22. Archibald JM, O'Kelly CJ, Doolittle WF. The chaperonin genes of jakobid and jakobid-like flagellates: Implications for eukaryotic evolution. Mol Biol Evol. 2002;19:422-431.

23. Loftus BJ, Fung E, Roncaglia P, et al. The genome of the basidiomycetous yeast and human pathogen Cryptococcus neoformans. Science. 2005;307:1321-1324.
24. Martinez D, Berka RM, Henrissat B, et al. Genome sequencing and analysis of the biomass-degrading fungus Trichoderma reesei (syn. Hypocrea jecorina). Nat Biotechnol. 2008;26:553-560.

25. Gudlaugsdottir S, Boswell DR, Wood GR, Ma J. Exon size distribution and the origin of introns. Genetica. 2007;131:299-306.

26. Saitou N, Nei M. The neighbor-joining method: A new method for reconstructing phylogenetic trees. Mol Biol Evol. 1987;4:406-425.

27. Ren XY, Vorst O, Fiers MWEJ, Stiekema WJ, Nap JP. In plants, highly expressed genes are the least compact. Trends Genet. 2006;22: $528-532$. 


\section{Supplementary tables}

Table SI Exonic chromosomal parameters of Plasmodium knowlesi

\begin{tabular}{|c|c|c|c|c|c|c|c|}
\hline Chromosome & $n_{\text {ex }}$ & $I_{e x}$ & $a_{\text {ex }}$ & $n l_{e x}$ & $L I_{e x}$ & $\mathrm{LO}_{\mathrm{ex}}$ & $P_{c}$ \\
\hline PKOI & $2.18 \pm 0.32$ & $2513 \pm 373$ & $|87| \pm 3||$ & $3.79 \pm 0.57$ & $2326 \pm 644$ & $265 I \pm 442$ & 42.29 \\
\hline PK02 & $2.61 \pm 0.39$ & $2234 \pm 384$ & $1476 \pm 340$ & $3.88 \pm 0.58$ & $199 \mid \pm 438$ & $254| \pm 67|$ & 55.84 \\
\hline PK03 & $2.74 \pm 0.36$ & $2313 \pm 346$ & $1455 \pm 252$ & $4.16 \pm 0.51$ & $2216 \pm 506$ & $2432 \pm 445$ & 55.10 \\
\hline PK04 & $2.70 \pm 0.38$ & $2159 \pm 291$ & $1436 \pm 263$ & $4.08 \pm 0.56$ & $1893 \pm 359$ & $2487 \pm 468$ & 55.17 \\
\hline PK05 & $2.75 \pm 0.38$ & $2048 \pm 304$ & $1387 \pm 277$ & $4.30 \pm 0.62$ & $1773 \pm 363$ & $2356 \pm 530$ & 52.90 \\
\hline PK06 & $2.67 \pm 0.38$ & $1996 \pm 284$ & $1308 \pm 205$ & $4.33 \pm 0.62$ & $1972 \pm 452$ & $2020 \pm 345$ & 50.00 \\
\hline PK07 & $2.65 \pm 0.28$ & $2093 \pm 218$ & $1396 \pm 189$ & $4.07 \pm 0.41$ & $1974 \pm 184$ & $2232 \pm 340$ & 53.85 \\
\hline PK08 & $2.47 \pm 0.23$ & $2062 \pm 216$ & $|45| \pm 193$ & $3.71 \pm 0.34$ & $1746 \pm 263$ & $2436 \pm 346$ & 54.18 \\
\hline PK09 & $2.69 \pm 0.24$ & $2226 \pm 203$ & $1492 \pm 175$ & $4.15 \pm 0.36$ & $2016 \pm 274$ & $2469 \pm 308$ & 53.64 \\
\hline PKIO & $2.43 \pm 0.24$ & $2114 \pm 305$ & $|43| \pm 205$ & $3.84 \pm 0.36$ & $2109 \pm 551$ & $2119 \pm 351$ & 50.32 \\
\hline PKII & $2.70 \pm 0.26$ & $2244 \pm 330$ & $1538 \pm 203$ & $4.44 \pm 0.41$ & $2025 \pm 288$ & $2459 \pm 345$ & 49.48 \\
\hline PKI2 & $2.59 \pm 0.20$ & $2213 \pm 187$ & $1483 \pm 145$ & $4.17 \pm 0.33$ & $2119 \pm 281$ & $2308 \pm 248$ & 50.29 \\
\hline PKI3 & $2.63 \pm 0.29$ & $2235 \pm 225$ & $1527 \pm 186$ & $4.30 \pm 0.49$ & $207 \mid \pm 323$ & $2395 \pm 311$ & 49.56 \\
\hline PKI4 & $2.42 \pm 0.19$ & $2195 \pm 166$ & $1542 \pm 155$ & $4.01 \pm 0.32$ & $2062 \pm 238$ & $2315 \pm 256$ & 47.36 \\
\hline Total & $2.59 \pm 0.07$ & $2185 \pm 66$ & $1487 \pm 55$ & $4.11 \pm 0.11$ & $2019 \pm 93$ & $2358 \pm 95$ & 51.43 \\
\hline
\end{tabular}

Table S2 Total variance explained

\begin{tabular}{lll}
\hline Component & \% of variance & Cumulative \% \\
\hline 1 & 61.413 & 61.413 \\
2 & 24.809 & 86.222 \\
3 & 8.290 & 94.512 \\
4 & 4.871 & 99.383 \\
\hline
\end{tabular}


Table S3 Component matrix extraction method: principal component analysis with four extracted components

\begin{tabular}{|c|c|c|c|c|c|c|}
\hline \multicolumn{7}{|c|}{ Component matrix (a) } \\
\hline \multirow[t]{2}{*}{ Abbr } & \multirow[t]{2}{*}{ Kingdom/supergroup } & \multirow[t]{2}{*}{ Organism } & \multicolumn{4}{|c|}{ Component } \\
\hline & & & $I$ & 2 & 3 & 4 \\
\hline$A D$ & Plantae & Arabidopsis thaliana & -0.928 & -0.314 & 0.069 & 0.172 \\
\hline $\mathrm{AF}$ & Fungi & Aspergillus fumigatus & -0.566 & 0.785 & -0.065 & 0.236 \\
\hline $\mathrm{BN}$ & Protista/Rhizaria & Bigelowiella natans & -0.601 & -0.545 & -0.210 & 0.544 \\
\hline CE & Animalia & Caenorhabditis elegans & -0.929 & -0.296 & 0.189 & -0.103 \\
\hline $\mathrm{CF}$ & Animalia & Canis familiaris & -0.956 & -0.240 & -0.025 & -0.119 \\
\hline CG & Fungi & Candida glabrata & 0.891 & -0.314 & -0.242 & -0.221 \\
\hline $\mathrm{CN}$ & Fungi & Cryptococcus neoformans & -0.986 & 0.040 & -0.123 & -0.103 \\
\hline $\mathrm{DH}$ & Fungi & Debaryomyces hansenii & 0.978 & -0.204 & 0.046 & -0.003 \\
\hline DM & Animalia & Drosophila melanogaster & -0.747 & 0.478 & 0.345 & -0.298 \\
\hline EC & Fungi & Encephalitozoon cuniculi & 0.582 & -0.805 & -0.082 & 0.085 \\
\hline EG & Fungi & Eremothecium gossypii & 0.946 & -0.250 & -0.137 & -0.152 \\
\hline GT & Protista/Chromista & Guillardia theta & 0.417 & -0.841 & 0.139 & 0.317 \\
\hline GZ & Fungi & Gibberella zeae & -0.555 & 0.769 & -0.276 & 0.096 \\
\hline $\mathrm{HA}$ & Protista/Chromista & Hemiselmis anderenii & 0.615 & -0.704 & -0.198 & -0.064 \\
\hline HS & Animalia & Homo sapiens & -0.967 & -0.229 & 0.050 & -0.091 \\
\hline KL & Fungi & Kluyveromyces lactis & 0.914 & -0.346 & -0.160 & -0.139 \\
\hline LB & Protista/Protozoa & Leishmania braziliensis & 0.677 & 0.628 & -0.380 & 0.020 \\
\hline MG & Fungi & Magnaporthe grisea & -0.776 & -0.492 & 0.237 & 0.314 \\
\hline MM & Animalia & Mus musculus & -0.965 & -0.255 & 0.037 & -0.038 \\
\hline MS & Plantae/Viridiplantae & Micromonas sp. RCC299 & 0.794 & 0.472 & 0.363 & 0.121 \\
\hline $\mathrm{NC}$ & Fungi & Neurospora crassa & -0.186 & 0.899 & -0.225 & 0.287 \\
\hline $\mathrm{OL}$ & Plantae/Viridiplantae & Ostreococcus lucimarinus & 0.737 & -0.030 & 0.542 & 0.400 \\
\hline OS & Plantae & Oryza sativa & -0.927 & -0.282 & 0.181 & 0.149 \\
\hline PF & Protista/Chromalveolata & Plasmodium falciparum & 0.629 & 0.685 & -0.327 & -0.168 \\
\hline PK & Protista/Chromalveolata & Plasmodium knowlesi & 0.646 & 0.632 & -0.396 & -0.152 \\
\hline PS & Fungi & Pichia stipitis & 0.752 & 0.468 & 0.439 & 0.136 \\
\hline PT & Protista/Chromalveolata & Paramecium tetraurelia & -0.706 & 0.642 & 0.179 & -0.239 \\
\hline SC & Fungi & Saccharomyces cerevisiaei & -0.969 & 0.119 & 0.215 & 0.026 \\
\hline SP & Fungi & Schizosaccharomyces pombe & 0.890 & 0.063 & -0.234 & 0.381 \\
\hline TA & Protista/Chromalveolata & Theileria annulata & -0.221 & 0.342 & -0.815 & 0.391 \\
\hline UM & Fungi & Ustilago maydis & 0.852 & 0.448 & -0.253 & -0.097 \\
\hline YL & Fungi & Yarrowia lipolytica & 0.862 & 0.296 & 0.390 & 0.130 \\
\hline
\end{tabular}


Table S4 Results obtained by k-means clustering technique and based on four principal components obtained by factor analysis of $A N_{e x}, A L_{e x}, A A_{e x}, A N I_{e x}, A L I_{e x}, A L O_{e x}$

\begin{tabular}{|c|c|c|c|c|c|c|c|c|c|c|c|c|c|c|c|c|c|c|c|}
\hline & 2 & 3 & 4 & 5 & 6 & 7 & 8 & 9 & 10 & 11 & 12 & 13 & 14 & 15 & 16 & 17 & 18 & 19 & 20 \\
\hline MG & I & 2 & 2 & 2 & 6 & 7 & 7 & 8 & 5 & 8 & I & 6 & 7 & 2 & 11 & 8 & 13 & 12 & 7 \\
\hline$A D$ & I & I & 3 & 5 & 4 & 3 & 2 & 9 & 3 & II & 6 & 2 & 6 & 11 & 16 & 9 & II & 11 & 8 \\
\hline OS & I & I & 3 & 5 & 4 & 3 & 2 & 9 & 3 & 11 & 6 & 2 & 6 & 11 & 16 & 9 & 11 & II & 8 \\
\hline HS & 1 & I & 3 & 5 & 4 & 3 & 2 & 9 & 3 & 11 & 9 & 8 & 4 & 10 & 8 & 15 & 16 & I & 5 \\
\hline $\mathrm{CF}$ & 1 & I & 3 & 5 & 4 & 3 & 2 & 9 & 3 & 11 & 9 & 8 & 4 & 10 & 8 & 15 & 16 & I & 5 \\
\hline MM & 1 & I & 3 & 5 & 4 & 3 & 2 & 9 & 3 & 11 & 9 & 8 & 4 & 10 & 8 & 15 & 16 & I & 5 \\
\hline CE & 1 & I & 3 & 5 & 4 & 3 & 2 & 9 & 3 & 11 & 8 & 8 & 5 & 10 & 8 & 15 & 16 & I & 14 \\
\hline $\mathrm{CN}$ & I & I & 3 & 5 & 4 & 3 & 2 & 9 & 3 & 11 & II & 8 & 11 & 10 & 15 & 16 & 8 & 10 & 19 \\
\hline $\mathrm{AF}$ & I & 2 & 2 & 2 & 5 & 4 & 3 & 2 & 8 & 5 & 5 & 10 & I & 3 & 14 & II & I & 3 & 4 \\
\hline GZ & I & 2 & 2 & 2 & 5 & 4 & 3 & 2 & 8 & 5 & 5 & 10 & I & 3 & 14 & 11 & I & 3 & 4 \\
\hline DM & I & 2 & 2 & 2 & 6 & 7 & 7 & 8 & 5 & 8 & I & 6 & 13 & 2 & 4 & 3 & 13 & 8 & 12 \\
\hline $\mathrm{NC}$ & 1 & 2 & 2 & 2 & 5 & 4 & 3 & 2 & 8 & 5 & 5 & 10 & I & 3 & 14 & 11 & I & 3 & 4 \\
\hline PT & 1 & 2 & 2 & 2 & 6 & 7 & 7 & 8 & 5 & 8 & I & 6 & 13 & 2 & 4 & 3 & 13 & 8 & 12 \\
\hline $\mathrm{BN}$ & 1 & I & 3 & 5 & 3 & 3 & 2 & 7 & 7 & 7 & 4 & 13 & 8 & 9 & 9 & 13 & 5 & 9 & 9 \\
\hline TA & I & 2 & 2 & 2 & 5 & 4 & 3 & 5 & 9 & 3 & 3 & 9 & 2 & 12 & 3 & 6 & 14 & 17 & 17 \\
\hline CG & 2 & 3 & I & 3 & 2 & 6 & 8 & I & 2 & 2 & 10 & 12 & 10 & 5 & 6 & I & 6 & 16 & 15 \\
\hline EG & 2 & 3 & I & 3 & 2 & 6 & 8 & I & 2 & 2 & 10 & 12 & 10 & 5 & 6 & I & 6 & 16 & 15 \\
\hline $\mathrm{KL}$ & 2 & 3 & I & 3 & 2 & 6 & 8 & I & 2 & 2 & 10 & 12 & 10 & 5 & 6 & I & 6 & 16 & 15 \\
\hline $\mathrm{DH}$ & 2 & 3 & I & 3 & 2 & 6 & 8 & I & 2 & 2 & 10 & 12 & 10 & 4 & 6 & 2 & 18 & 5 & 18 \\
\hline EC & 2 & 3 & I & 3 & 2 & 5 & 4 & 6 & 10 & 9 & 2 & 5 & 3 & 14 & 2 & 12 & 7 & 14 & 10 \\
\hline GT & 2 & 3 & I & 3 & 2 & 5 & 4 & 6 & 10 & 10 & 2 & 5 & 3 & 14 & 2 & 17 & 4 & 14 & 6 \\
\hline $\mathrm{HA}$ & 2 & 3 & I & 3 & 2 & 5 & 4 & 6 & 10 & 9 & 2 & 5 & 3 & 14 & 2 & 12 & 7 & 14 & 10 \\
\hline LB & 2 & 3 & 4 & 4 & I & I & 1 & 3 & I & I & 12 & 4 & 14 & 7 & 10 & 4 & 3 & 2 & 3 \\
\hline MS & 2 & 3 & 4 & 4 & I & I & I & 3 & I & 1 & 12 & 4 & 14 & 15 & 10 & 4 & 15 & 18 & 20 \\
\hline PF & 2 & 3 & 4 & I & I & 2 & 6 & 4 & 4 & 4 & 7 & 3 & 12 & I & 5 & 10 & 10 & 15 & 2 \\
\hline PK & 2 & 3 & 4 & I & I & 2 & 6 & 4 & 4 & 4 & 7 & 3 & 12 & I & 7 & 10 & 10 & 15 & 2 \\
\hline UM & 2 & 3 & 4 & I & I & 2 & 6 & 4 & 4 & 4 & 7 & 3 & 12 & I & 12 & 10 & 2 & 15 & 13 \\
\hline PS & 2 & 3 & 4 & 4 & I & I & 1 & 3 & I & 1 & 12 & 4 & 14 & 15 & 10 & 4 & 15 & 19 & 20 \\
\hline SC & 2 & 3 & 4 & 4 & I & I & 5 & 3 & 6 & 1 & 12 & 7 & 9 & 6 & 10 & 7 & 17 & 6 & 16 \\
\hline YL & 2 & 3 & 4 & 4 & I & I & 1 & 3 & 6 & 1 & 12 & 7 & 14 & 15 & 10 & 7 & 15 & 4 & 20 \\
\hline SP & 2 & 3 & 4 & I & I & 6 & 5 & I & 2 & 6 & 10 & I & 9 & 8 & I & 5 & 12 & 7 & I \\
\hline OL & 2 & 3 & 4 & 4 & I & I & 5 & 3 & 6 & 1 & 12 & 11 & 9 & 13 & 13 & 14 & 9 & 13 & 11 \\
\hline
\end{tabular}


Table S5 Results obtained by partitioning around medoids clustering technique and based on four principal components obtained by factor analysis of $A N_{e x}, A L_{e x}, A A_{e x}, A N I_{e x}, A L I_{e x}, A L O_{e x}$

\begin{tabular}{|c|c|c|c|c|c|c|c|c|c|c|c|c|c|c|c|c|c|c|c|}
\hline & 2 & 3 & 4 & 5 & 6 & 7 & 8 & 9 & 10 & 11 & 12 & 13 & 14 & 15 & 16 & 17 & 18 & 19 & 20 \\
\hline MG & 2 & 3 & 3 & 3 & 3 & 3 & 3 & 3 & 3 & 3 & 12 & 12 & 12 & 12 & 12 & 12 & 12 & 12 & 12 \\
\hline$A D$ & 2 & 2 & 2 & 2 & 2 & 2 & 2 & 2 & 2 & 2 & 2 & 2 & 2 & 2 & 16 & 16 & 16 & 16 & 16 \\
\hline OS & 2 & 2 & 2 & 2 & 2 & 2 & 2 & 2 & 2 & 2 & 2 & 2 & 2 & 2 & 16 & 16 & 16 & 16 & 16 \\
\hline HS & 2 & 2 & 2 & 2 & 2 & 2 & 2 & 2 & 2 & 2 & 2 & 2 & 2 & 2 & 2 & 2 & 2 & 2 & 2 \\
\hline $\mathrm{CF}$ & 2 & 2 & 2 & 2 & 2 & 2 & 2 & 2 & 2 & 2 & 2 & 2 & 2 & 2 & 2 & 2 & 2 & 2 & 2 \\
\hline MM & 2 & 2 & 2 & 2 & 2 & 2 & 2 & 2 & 2 & 2 & 2 & 2 & 2 & 2 & 2 & 2 & 2 & 2 & 2 \\
\hline$C E$ & 2 & 2 & 2 & 2 & 2 & 2 & 2 & 2 & 2 & 2 & 2 & 2 & 2 & 2 & 2 & 2 & 2 & 2 & 2 \\
\hline $\mathrm{CN}$ & 2 & 2 & 2 & 2 & 2 & 2 & 2 & 2 & 2 & 2 & 2 & 2 & 2 & 2 & 2 & 17 & 17 & 17 & 17 \\
\hline $\mathrm{AF}$ & 2 & 3 & 3 & 3 & 3 & 3 & 3 & 3 & 3 & 3 & 3 & 3 & 3 & 3 & 3 & 3 & 3 & 3 & 3 \\
\hline GZ & 2 & 3 & 3 & 3 & 3 & 3 & 3 & 3 & 3 & 3 & 3 & 3 & 3 & 3 & 3 & 3 & 3 & 3 & 20 \\
\hline NC & 2 & 3 & 3 & 3 & 3 & 3 & 3 & 3 & 3 & 3 & 3 & 3 & 14 & 14 & 14 & 14 & 14 & 14 & 14 \\
\hline CG & I & I & 4 & 4 & 4 & 4 & 4 & 4 & 4 & 4 & 4 & 4 & 4 & 4 & 4 & 4 & 4 & 4 & 4 \\
\hline EG & I & I & 4 & 4 & 4 & 4 & 4 & 4 & 4 & 4 & 4 & 4 & 4 & 4 & 4 & 4 & 4 & 4 & 4 \\
\hline $\mathrm{KL}$ & I & I & 4 & 4 & 4 & 4 & 4 & 4 & 4 & 4 & 4 & 4 & 4 & 4 & 4 & 4 & 4 & 4 & 4 \\
\hline $\mathrm{DH}$ & I & I & 4 & 4 & 4 & 4 & 4 & 4 & 4 & 4 & 4 & 4 & 4 & 4 & 4 & 4 & 4 & 19 & 19 \\
\hline PF & I & I & I & 5 & 5 & 5 & 5 & 5 & 5 & 5 & 5 & 5 & 5 & 5 & 5 & 5 & 5 & 5 & 5 \\
\hline PK & I & I & 1 & 5 & 5 & 5 & 5 & 5 & 5 & 5 & 5 & 5 & 5 & 5 & 5 & 5 & 5 & 5 & 5 \\
\hline UM & I & I & I & 5 & 5 & 5 & 5 & 5 & 5 & 5 & 5 & 5 & 5 & 5 & 5 & 5 & 18 & 18 & 18 \\
\hline EC & I & I & 4 & 4 & 6 & 6 & 6 & 6 & 6 & 6 & 6 & 6 & 6 & 6 & 6 & 6 & 6 & 6 & 6 \\
\hline GT & I & I & 4 & 4 & 6 & 6 & 6 & 6 & 6 & 6 & 6 & 6 & 6 & 15 & 15 & 15 & 15 & 15 & 15 \\
\hline HA & I & I & 4 & 4 & 6 & 6 & 6 & 6 & 6 & 6 & 6 & 6 & 6 & 6 & 6 & 6 & 6 & 6 & 6 \\
\hline LB & I & I & I & I & I & I & I & I & I & I & 1 & 1 & 1 & I & I & 1 & I & I & I \\
\hline MS & I & I & I & I & I & I & I & I & I & I & 1 & I & I & I & I & I & I & I & I \\
\hline PS & I & I & I & I & I & I & I & I & I & I & 1 & 1 & 1 & I & I & I & I & I & I \\
\hline SC & I & I & I & I & I & I & I & I & I & 1 & 1 & 13 & 13 & 13 & 13 & 13 & 13 & 13 & 13 \\
\hline YL & I & I & I & I & I & I & I & I & I & I & 1 & 1 & I & I & I & I & I & I & I \\
\hline DM & 2 & 3 & 3 & 3 & 3 & 7 & 8 & 8 & 8 & 8 & 8 & 8 & 8 & 8 & 8 & 8 & 8 & 8 & 8 \\
\hline PT & 2 & 3 & 3 & 3 & 3 & 7 & 8 & 8 & 8 & 8 & 8 & 8 & 8 & 8 & 8 & 8 & 8 & 8 & 8 \\
\hline TA & 2 & 3 & 3 & 3 & 3 & 3 & 7 & 9 & 9 & 9 & 9 & 9 & 9 & 9 & 9 & 9 & 9 & 9 & 9 \\
\hline $\mathrm{BN}$ & 2 & 2 & 2 & 2 & 2 & 2 & 2 & 7 & 7 & 7 & 7 & 7 & 7 & 7 & 7 & 7 & 7 & 7 & 7 \\
\hline SP & I & I & 4 & 4 & 4 & 4 & 4 & 4 & 10 & 10 & 10 & 10 & 10 & 10 & 10 & 10 & 10 & 10 & 10 \\
\hline OL & I & I & I & I & 1 & I & I & 1 & I & 11 & 11 & 11 & 11 & II & 11 & 11 & II & II & 11 \\
\hline
\end{tabular}

\section{Publish your work in this journal}

Open Access Bioinformatics is an international, peer-reviewed, open access journal publishing original research, reports, reviews and commentaries on all areas of bioinformatics. The manuscript management system is completely online and includes a very quick and fair peer-review system. Visit http://www.dovepress.com/testimonials.php to read real quotes from published authors. 